\title{
Analysis of the reasons for nurses' confusion in relation to the concept of brain death from clinical and legal points of view
}

\author{
Hamideh Yazdi Moghaddam ${ }^{1}$, Alireza Pouresmaeili², Zahra Sadat Manzari ${ }^{3}$
}

${ }^{1}$ Ph.D. in Nursing, Faculty of Paramedical, Sabzevar University of Medical Sciences, Sabzevar, Iran

${ }^{2}$ Ph.D. in Private Law, Assistant Professor, Department of Law, Faculty of Theology and Islamic Sciences, Hakim Sabzevari University, Sabzevar, Iran

${ }^{3}$ Ph.D. of Nursing, Assistant Professor, Department of Medical-surgical nursing, Faculty of Nursing and Midwifery, Mashhad University of Medical Sciences, Mashhad, Iran

Type of article: Review

\begin{abstract}
Background: Nurses in intensive care units (ICU) play a key role in taking care of brain dead patients and they are often in contact with such patients given the high rate of brain deaths. Consequently, they are in a challenging and stressful condition, which may be aggravated due to the need for increased organ donation, and this may also affect the quality of care provided for these patients.

Objective: This review analyzes the different studies on the concept of brain death from both medical and legal points of view. The aim of this study was to identify the challenges the nurses face with the perception of the concept of brain death and its effects on the care provided for the patients suffering from brain death, as well as organ donation.

Methods: This Narrative review study summarizes the information obtained from relevant literature through a targeted search of library texts and electronic resources including Science Direct, PubMed, Google Scholar, Scientific Information Database (SID), IranMedex, and Magiran databases during 2000-2017 in English and Persian, on the subject of interest by keywords such as brain death, organ donation, care, legal, regulations and nursing. Finally, a total of 44 relevant articles were reviewed.

Results: Nurses play an important role in taking care of brain dead patients; therefore, recognizing challenges can be the first step in taking accurate care of these patients and subsequently maintaining the organs' health for transplants. Although there are clinical and legal uncertainties regarding the concept of brain death, nurses have an important effect on taking care of brain dead patients as well as the rate of organ donation.

Conclusion: Nurses in intensive care units should have a clear understanding of the concept of brain death in order to be able to take care of brain dead patients by avoiding psychological effects. In this regard, it is recommended that a comprehensive educational program be designed on the dimensions of brain death. Therefore, the nurses' awareness of brain death and their ability to clarify this concept to the patients' relatives would have a great effect on the decrease in the challenges and the stress imposed on the nurses. Also, there would be an increase in the quality of the care given to brain dead patients and even the rate of organ donation.
\end{abstract}

Keywords: Brain death, Organ donation, Nurse, Care, Legal

\section{Introduction}

Taking care of dying patients is one of the important aspects of nursing care. Brain death is considered as a type of death (1) and is one of the main concepts in nursing theories (2). As a result, nurses and especially nurses of the intensive care units, as one of the largest groups among health care providers, play a critical role in taking care of

\section{Corresponding author:}

Assistant Professor Dr. Zahra Sadat Manzari, Department of Medical-surgical nursing, Faculty of Nursing and Midwifery, Mashhad University of Medical Sciences, Mashhad, Iran.

Tel:+98.5138598019, Fax:+98.5138597313, Email: Manzariz@mums.ac.ir

Received: August 13, 2017, Accepted: September 26, 2017, Published: May 2018

iThenticate screening: September 28, 2017, English editing: April 14, 2018, Quality control: April 26, 2018

This article has been reviewed / commented by five experts

(C) 2018 The Authors. This is an open access article under the terms of the Creative Commons Attribution-NonCommercialNoDerivs License, which permits use and distribution in any medium, provided the original work is properly cited, the use is non-commercial and no modifications or adaptations are made. 
brain dead patients and are mostly in contact with such patients (3). According to the statistics, $2-3 \%$ of deaths are observed in public hospitals and $12-14 \%$ of deaths occur in brain intensive care units (4). Recently, researchers have become interested in the experiences of nurses caring dying. A nurse is often the first person who starts to determine the potentiality of a patient for being an organ donor since s/he can check clinical signs of decompensation. When a person is declared brain dead, it is the nurse who maintains hemodynamic stability so that donor organs remain viable (5). It is not professional for nurses to accept the role without satisfying themselves that the concept of brain death is coherent and robust. Instead, they should critically examine the definition of death currently informing clinical practice (6). Since the brain death concept has been misinterpreted repeatedly among respondents, the nurses' attitudes toward transplantation have been negatively affected (7). Accordingly, the nurses of the intensive care units face a lot of uncertainties about brain death which affect the psychological health of nurses and the quality of care provided for brain death patients at a micro level as well as the rate of organ donation at a macro level (8). The most significant obscurity for nurses is the concept of brain death, which has some other consequences. Research has shown that nurses do not have the necessary knowledge about brain death or that they acquire their knowledge through experience and need training in terms of brain death and organ donation rules (9). Improving the nurses' knowledge about brain death can dramatically contribute to the transfer of one life to one or more individuals (10). By reason of the ICU nurses appropriately understanding the concept of brain death, they as those who, after physicians, are mostly in contact with the brain death patients and their families, can explain the status of the brain death patients to their families in simple terms (11). In addition, ICU nurses, as people who spend most of their time at the patient's bedside, help the brain dead patient's family to accept the patient's circumstances. On the other hand, if the nurses manage the conditions well, the family may agree to donate the patient's organs. Furthermore, a nurse can also play a role in alleviating the suffering of the patient's family, so the quality of comprehensive nursing for brain dead patients depends on understanding the concept of brain death and accepting it (12). Therefore, the nurse's exposure to such a situation, namely providing the correct care for the patient, explaining brain death to the patient's family, relieving them of any misgivings and helping them to decide on organ donation, places the nurse in a stressful status that requires the nurse's awareness of the entire care process for the brain dead patient. This makes planning to support nurses more tangible (13).

A close look at Iran regulations and rules about brain death clarifies that the concept of brain death and the nurses' uncertainties about the concept of brain death are, to a small extent, caused by ambiguity of the relevant regulations. Although the "Act of transplantation for deceased patients or patients whose brain death is certain" (hereafter, the Organ Transplantation Brain Death Act) was passed in 2000 and organ transplantation was not also forbidden in Iran $(14,15)$. Studies have indicated that only half of physicians and nurses and general practitioners have sufficient knowledge about the concept of brain death, organ donation and its rules and that there is a significant relationship between their knowledge and their positive attitudes towards organ donation (16). In addition to legal issues in this regard, ICU nurses do not attempt to encourage organ transplants due to their fear of enhancing the psychological pressure on the patient's family members (17). With regard to the centrality of this issue in terms of the culture and religion of each society, the way nurses face this problem is of importance. Therefore, The ICU nurses should be aware of medical, moral and legal questions in dealing with this situation and understand the concept of brain death well, because ignoring these issues would have unintended effects on nurses and patients and can affect the quality of care for the brain dead patients, which plays an important role in improving organ donation rate. At the end, through a narrative review, this study attempted to identify and evaluate the challenges the nurses face in dealing with the concept of brain death from clinical and legal points of view.

\section{Material and Methods}

This study is a narrative review aiming to investigate the challenges the nurses face in dealing with the concept of brain death from a clinical and legal point of view in providing care for brain dead patients. In the present narrative review, scientific databases including PubMed, Science Direct, Google Scholar, SID, IranMedex, and Magiran were used to search for relevant literature. The primary search-targeted articles were published in English and Persian from 2000-2017 on the subject of interest by keywords including brain death, organ donation, care, legal, regulations and nursing, whether in title or abstract. In addition, the references of selected papers were also searched. In the first section of this study, the questions were designed and formulated. The main question was presented as: "Based on the results of studies, what are the challenges nurses may face regarding brain death while providing care to brain dead patients? The inclusion criteria constituted the conditions such that trial data has to be obtained from the original articles rather than the results of the reviews, and 'evaluation of the nursing challenges of patients with brain death' has to be considered the main objective of the study; moreover, the challenges have to be associated with critical care nursing. A point that should be clarified here is that, all the reviewed articles were published in 
English and Persian. To determine which documentation to use, first the titles found by the search engine were carefully looked into to make sure they were related to the field. Then the issues were categorized into two groups of the definition of brain death from medical or clinical and legal points of view. Then the challenges the nurses faced on the concept of brain death were investigated. Also, after an investigation of the titles of the articles, there was an evaluation of the relation between the abstract and the aim of the paper. The selected materials were all studied carefully in detail. In this method, to prevent browser bias, all articles and abstracts were accordingly examined by two reviewers independently while there was an emphasis on the importance of entrance criteria. Studies with similar titles but varied subject matters were excluded. Finally, 44 studies were selected and approved.

\section{Results}

This study concentrated on a narrative examination of 41 papers dealing with challenges facing nurses in the concept of brain death. As the findings indicated, one reason for the ambiguity in understanding the concept of brain death is the various definitions offered for this term. Although more than two decades have passed since the approval of the transplant law, many nurses and caregivers still do not recognize the concept of brain death appropriately. In other words, there are different definitions for brain death in different texts such as medical or legal texts, which aggravates the nurses' ambiguity in taking care of brain dead patients. Although brain death is thought of as a misleading term which includes different kinds of death such as deep coma, it should only be defined as irreversible brain cease (18). So, this paper investigates different studies on the concept of brain death from both medical and legal points of view and then analyzes the challenges the nurses face in understanding the concept of brain death and its effect on the care given to brain dead patients as well as organ donation.

\subsection{Definition of brain death from clinical point of view}

Death is an incident that is not easy to define (19). Today, the definition of death presented by the community of physicians, especially neurologists, has dramatically changed (20). In modern medicine, it is believed that brain health is a prerequisite in order for a person to be alive and recover (21). Until 1959, cardiac and respiratory arrest was accepted as death with no doubt and uncertainty. Following a change in technology, the brain death concept was redefined (22). Nowadays, cardiovascular system arrest and failure is no longer considered as an irreversible condition concerning the advances in resuscitation equipment and intensive care technology (23). Hence, brain death is the result of advancement and technology. It should also be noted that brain death is a conversational term to refer to an irreversible brain arrest. In addition, brain death is a misleading term, which includes different types of death and deep coma; however, brain death should only be regarded as an irreversible brain arrest (18). There are some controversies regarding the definitions of brain death presented in various texts. In this regard, three theories are put forth.

\subsubsection{Whole brain death:}

From this perspective, the irreversible arrest of a vital organ's function as a whole, is considered as brain death. According to this definition, the vital functions refer to actions such as breathing and consciousness, upon which one's health and survival depends $(1,18)$. Accordingly, the basic and essential criterion to diagnose death is as follows: Permanent cessation and interruption of all clinical functions of the whole brain (18). Thus, this definition is based on the traditional definition of death and includes the cessation of the blood flow as well as the arrest of vital and essential functions (24). In addition to vital functions, the coordinating role of the brain in the circulation of blood is also considered (25). The strengths of this definition, in contrast to other definitions, are as follows: (a) loyalty to the traditional concepts of death and attention to the role of the brain in blood circulation; (b) declaration of a single criterion for the diagnoses of brain death to eliminate the suspicions regarding the death or life of an individual; and (c) declaring a single criterion makes the patients' families consider brain death as irreversible and, consequently, be prepared for organ donation or burial. From a legal perspective, this situation is also in effect and the heirs can apply for the estate of the deceased (24). Two main foundations in this viewpoint are: a) Brain is the "core organ" that separates human life from its personality and identity since, contrary to the traditional notion that regards heart arrest as an equivalent to death, the cessation of the brain function is now the current sense of death. b) The heart plays a biological role in the survival of human life. The heart, as the center of the body, consisting of the upper and lower parts, is responsible for coordinating the vital organs of the body. With the cessation of the heart function, the command center collapses and the coordination of the members is also lost (26). This view is accepted in the United States, Canada and many other countries and is more common in comparison to the other two theories. The Uniform Determination of Death Act (UDDA) that was approved by the American Medical Association and the American Bar Association states that: "An individual who has sustained either (1) irreversible cessation of circulatory and respiratory functions, or (2) irreversible cessation of all functions of the entire brain, including the brain stem, is dead. A determination of death must be made in accordance with accepted medical standards" (26). In 
all Canadian provinces and territories, the legal definition of brain death is "according to accepted medical practice" (27). Concerning the irreversible cessation of circulation and respiratory function, the law remains faithful to the traditional sense of death, i.e. cardiac and pulmonary arrest, and adds a criterion indicating that brain death should be determined in accordance with accepted medical standards to expand and standardize the representation of the brain deaths (26). Neurological examination is one of the clinical standards for the diagnosis of brain death in many countries, which should be performed carefully. This study consists of three topics: Coma, lack of brain stem reflexes and apnea. The most important criteria to diagnose brain death are the lack of motor response, lack of pupil response to light, lack of corneal reflexes, lack of gag reflex, lack of ocular-atrial response (caloric test), lack of coughing in the case of endotracheal suction and lack of sucking and routing reflex. In addition, auxiliary diagnostic procedures including radionuclide scan and brain death angiography are used to indicate the absence of brain blood flow (28). Furthermore, laboratory tests that are usually performed after six hours of initial clinical examination of death are also run to confirm brain death. These tests include Angiography, Electroencephalography, CT scan, Doppler ultrasound and somatosensory stimulation potential (3).

3.1.2. Brain stem death:

The advocates of this theory believe that the cerebral cortex also dies with the death of the brain stem (1). This view is accepted in the UK and reflected in the code of practice for the diagnosis and confirmation of death (29). The proponents of this view have provided three foundations to confirm this theory: (a) Awareness is necessary for life; (b) Death is a process not an incident or a sudden incident; and (c) The death of a living organ is sufficient to represent death; therefore, the death of all organs and general death is not necessary (1).

\subsubsection{Higher brain death:}

In contrast to the previous viewpoints, this view is only accepted by a group of academic scholars, but has not been endorsed by any medical societies, and forms the basis of no law in any country or jurisdiction. According to this theory, the loss of a part of the brain that leads to a lack of consciousness should also be considered as death and this is the point differentiating this theory from the first theory. Thus, key brain functions such as memory, consciousness and personality also appear in the upper part of the brain and these activities form a living individual. As a result, the loss of this part amounts to the death of an individual. Sarbey briefly describes this viewpoint as follows: Death is the irreversible loss of that which is essentially significant to the nature of human (24). As a result, brain death could be defined as irreversible cessation of consciousness. In summary, the logical analogy used by the followers of this theory can be defined as follows: The loss of consciousness requires the elimination of the essential components of life (minor analogy) and the elimination of the essential components of human life also requires death (major analogy), and consequently, the loss of consciousness is equivalent to an individual's death (29).

\subsection{Brain death from legal point of view}

The first living-related kidney transplantation in the Middle East was performed at Shiraz University Hospital in 1968. In 2000, after two previous rejections, "the Act of transplantation for deceased patients or a patient whose brain death is certain" (the Brain Death and Organ Transplantation Act) was passed by the Iranian parliament, legalizing organ transplantation from deceased donors and its 'Rules of Procedure' was passed in 2002. After this law was passed, the Iranian Ministry of Health began establishing Brain Death Identification Units and Organ Procurement Centers throughout Iran. The Brain Death and Organ Transplantation Act mandated that all organ transplants be performed only at university hospitals approved by the Ministry of Health (24). According to article 1 of the Rules of Procedure, "brain Death is the irreversible discontinuation of all cortical (cerebral cortex), subcortical (subcortical layer) and brain stem functions." The conditions for brain death and its criteria and standards are also subject to specific guidelines (30). The definition of brain death in Iran is rooted in the first brain death theory and is the same as the definition given in the United States of America. The difference is that the traditional criteria and standard for irreversible cessation of circulation and respiratory function are presented along with the concept of brain death in the United States. The second difference is that the legislators in the United States prescribe brain death according to accepted medical standards and this requirement is not observed in Iran's regulations and the authentication conditions are referred to specific guidelines. The US legislator's reference to the cessation of blood circulation and respiratory function can also be addressed in Iran because individuals whose brain death is assumed to be certain according to the custom have a plant life and are not considered as dead. Subsequently, any action leading to the termination of their lives is not permissible and may be regarded as an act of manslaughter (31). Therefore, it would have been better if the conventional and traditional standard of death, namely the cessation of cardiovascular and respiratory function, had been carefully considered so that the patient could be considered as dead by cardiac and pulmonary arrest. It should be noted that plant life and coma are different from brain death because the person in a coma would either recover, his consciousness level approaching normal, or else his health status would deteriorate and he would die. Today, brain death is absolute in terms of medicine and this person would 
not be alive again. There is still the living potential and power in plant life (21). Other challenges that exist in law in terms of brain death and consequently may be a hindrance to the organ donation are listed below:

3.2.1. Identification of the patient's family and obtaining their written consent to donate:

In cases of death, the patient's body organs would be donated under two circumstances: a) The patient's will regarding the donation; b) His/her family's consent to donate. (Articles 5-7 of the Rules of Procedure). According to the Rules of Procedure, the guardians of the deceased are his adult legal descendants who can announce their consent to the transplantation of the organs. Obtaining the consent of all mentioned descendants is obligatory (Article 7 of the Rules of Procedure). Therefore, the Brain Death and Organ Transplantation Act considers the deceased's guardians as his adult heirs, all of whom should agree and the identification of heirs is a specialized and difficult task. In addition, the following criticisms can be made:

1) Given that all the heirs of a brain dead patient should consent to the donation, if some heirs do not consent, should the donation be performed on the basis of the heirs' consent?

2) What action should be taken if the immediate family or heirs of the patient are unknown or some of his/her heirs are unavailable?

3) If the brain death patient has no immediate family or heir, can the ruler of the community allow the donation? It should be noted that the deceased has no heir in discussing the inheritance.

4) Who should give consent if the immediate family of the brain death patient is in a coma? Suppose a husband and his wife are sent to a hospital after a car accident, in which case the brain death of the woman is certain and the husband is in a coma. The only heir of the husband who is in a coma is his father. Who has authority to give consent to organ donation in this situation? Further, if the husband's father is in a mental institution, who should consent in this situation?

3.2.2. Consensus or non-consensus of transplantation team members:

According to the Organ Transplantation Brain Death Act, brain death must be diagnosed and certified by 4 physicians, including a neurologist, a neurosurgeon, an internist, and an anesthesiologist, none of whom can be part of a transplantation team and each of whom should examine the patient separately. The rules do not specify whether or not the consensus among the four members of the medical team is of essence and whose comments are preferred in the case of discrepancy among the members.

\subsection{The challenges faced by the nurses on the perception of the concept of brain death}

Understanding the concept of brain death and taking care of brain dead patients is considered as a crucial part of nursing function in intensive care unit (32). Although the community of health care providers, especially nurses, is expected to have clearly learned and accepted the concept of brain death, the findings reveal that this concept has not been accepted among medical and nursing professions and indeed, it is of contradictory functions. According to Coyle, it is considerably difficult for most nurses to perceive the concept of brain death and it challenges all their previous beliefs on death (11), since the concepts and perceptions related to brain death go beyond understanding natural death. According to Pearson, there is a direct relationship between the uncertainties and contradiction about the concept of brain death and ambiguity in the concept of death for the nurses working in intensive care units (13). The reason behind it is that brain death has been defined as real death in which there is no hope for recovery. However, the physical appearance of the brain dead patient, the presence of his/her family members, and the performance of the nurses imply that he/she is still alive, which all aggravate the sense of ambiguity. So, the perception of the concept of brain death is considerably challenging to these nurses and the contradiction faced by the nurses is not based on logic, but is completely emotional and based on experience (13). According to the studies done by Ronayne, despite the findings of the science of physiology on brain death, the experience the nurses gain in taking care of these patients imposes a lot of stress on them for a long period of time because understanding the physiology of the death of these patients is in contradiction to their appearance, body temperature and heartbeat (3). The reason behind it is that there is no change in the patients' appearance although they have been diagnosed with brain death. This increases levels of stress in the nurses responsible for their care and leads to problems for nurses in the perception of diagnosing brain death (3). In a study by Keshtkaran et al., there was a lack of confidence in the diagnosis and confirmation of brain death among the nurses (33). So, the nurses were in a particular kind of uncertainty and doubt in exposing and taking care of brain death organ donor patients, which was also evident in the interaction with the patients' families. Due to their hope for the patient's recovery, the nurses would not declare the patient's brain death to their family. Such confusion and doubt made the nurses unhappy and reinforced the denial of the patients' death by their families (33). Studies conducted by other researchers such as Floden et al. (19), Marck et al. (34), Pearson et al. (13) and Salahuddin (28) also revealed a degree of ambiguity and uncertainty in terms of diagnosing brain death. Thus, diagnosing and confirming brain death is considered as the greatest concern and stressor for nurses (3). Miller et al. found that nurses were afraid of facing brain dead patients due to their suspicion 
and uncertainty about their death, since they did not have sufficient knowledge regarding the diagnostic tests of brain death and its process (20). The Findings of a study by Mirhashemi suggested that the ICU nurses had some concerns about issues such as brain death diagnostic tests and mental perceptions regarding donation and transplant process, which made them suffer from anxiety and suspicion (23). Another study also confirmed that less than half of the nurses are confident in the diagnosis of brain death (19). Kima and Eliot's study in South Korea also showed that individuals' attitudes, beliefs and behaviors are influenced by their cultural background, social norms and religious beliefs. These items were classified as religious, cultural, social, educational and empirical factors (35). However, it should be noted that the ambiguities that the nurses face in relation to the perception of the concept of brain death, as it was discussed several times in the studies carried out, is due to lack of a clear definition of brain death as well as inadequate education among nurses. These ambiguities in relation to the concept of brain death and also the challenges faced by the nurses, could affect the amount of organ donations.

\section{Discussion}

Studies have shown that it is considerably difficult and stressful for nurses to face and take care of brain dead patients, because they are compelled to go through unreasonable pressure and stress in taking care of these patients while facing different ambiguities and conflicts in this regard (18). Based on the studies, none of the doctors and nurses can easily get along with brain death diagnosis (9). The study by Floden indicated that only the nurses working in hospital wards other than ICU were expected to face more ambiguity and uncertainty on the concept of brain death and whether the brain dead patient were dead or not (36). However, it transpired that the nurses working in ICU also suffer from this sense of uncertainty. The reason behind it is that they are of little knowledge on the concept of brain death and think of the brain dead patient as a living individual (38). Cohen argued that confusion and uncertainty on confirming brain death and different concepts of death among health care professions may lead to adverse consequences in the process of providing care for organ donors (37). According to Rittner et al. (2003), the sense of ambiguity and uncertainty among nurses may result from professional challenges in ICU because the nurse is taking care of a patient who seems to be alive (38). However he is indeed dead, but has some living organs which could save the lives of other patients. So, taking care of these patients is actually in vain and this issue presents a challenge to the nurses and develops into a kind of confusion, doubt or uncertainty. There would never be an answer to this question and the nurse is left confounded as the patient's heart is still beating, and there is a failure in understanding whether the patient is dead or not (38). Therefore, the nurses' awareness of brain death and their ability to clarify this concept to the patients' families would have a great effect on the decrease in the challenges and the stress imposed on them. According to the studies done in Iran and the large number of brain deaths, only half of the doctors and nurses have sufficient knowledge about organ donation and its related rules and there is a meaningful relationship between knowledge and a positive attitude toward organ donation $(16,39)$.

According to Ozdag, nurses acquire their knowledge through experience and need training in the field of brain death and organ donation regulations (9). Hence, adequate knowledge and education levels are considered as factors affecting admission and tendency for organ donation (9). Therefore, nurses and transplant personnel need specialized and legal education in terms of all aspects of brain death, including understanding the concept of brain death, encountering organ donation and supporting the families of brain dead patients. In Iran, such an educational content covering various aspects of this subject has not been prepared for the treatment staff because the care process involves an interactive process, and interaction is the main means for establishing a reliable communication and examining the resources and needs of the patient and essentially the major tool for nursing interventions (40). On the other hand, the transplant team is also in charge of obtaining the satisfaction and consent of the patient's families and carrying out the related legal tasks. Consequently, prolonging this process can lead to death or loss of vital organs due to the specific and vulnerable conditions of the brain dead patient $(30,31)$. Now, given that the process of taking care of brain dead patients until the organ donation phase is team-oriented and requires the cooperation of physicians, nurses in the diagnoses and transplantation centers and the members of the transplant team, the nurses play an important role in this process due to their continuous presence alongside the patient and the numerous referrals of the patient's family to them and by reason of them being the first persons to observe the clinical symptoms of brain death in a patient and inform the doctor. Thus, understanding the brain death concept reduces the nurses' stress and psychological effects and thus improves the quality of care provided for the patients. In addition to the lack of plans to promote nurses' awareness, some ambiguities in the regulations also lead to a lack of understanding of brain death for nurses and may, at a large scale, be one of the reasons for reducing the rate of organ donation. Therefore, supplying nurses with medical, clinical and legal training issues regarding brain death can be very helpful in the quality of care for brain death patients and accelerate the donation process. 


\section{Conclusions}

The results of this study revealed that nurses face challenges regarding the perception of the concept of brain death from both clinical and legal points of view. Therefore, because nurses have a key role in taking care of brain dead patients in a health care team, means and methods on how to remove these challenges should be instigated. These findings are of highly practical importance as time is a determining factor in saving the patients' living organs to donate. Thus, if the patients are not properly taken care of, there will be negative effects on the quality of the organs which are to be donated. So, understanding the concept of brain death by nurses will have a great effect on providing brain dead patients with care and also the relationship with the patients' family to give consent to organ donation. Therefore, it is recommended that university education systems and hospitals provide special educational and support programs on all aspects of brain death and donations from university level in order to improve the nurses' knowledge on the process of brain death and organ donation, while taking into account their psychological and emotional needs. Taking into account these findings is essential to maintaining nursing health, increasing the quality and safety of nursing care and the health of vital organs on brain death donors and enhancing the rate of transplantation. Also, further research could be administered on the use of educational methods and supportive models in relation to the perception of the concept of brain death and the process of taking care of patients, for ICU nurses.

\section{Acknowledgments:}

The authors hereby express their gratitude to the authorities of Mashhad University of Medical Sciences for sponsoring the study, and the authorities of the Faculty of Nursing and Midwifery for their valuable support.

\section{Conflict of Interest:}

There is no conflict of interest to be declared.

\section{Authors' contributions:}

All authors contributed to this project and article equally. All authors read and approved the final manuscript.

\section{References:}

1) Bosnell R, Madder H. Concepts of brain death. Surgery. 2011; 29(7): 289-94. doi: 10.1016/j.mpsur.2011.04.001.

2) Motevalizade S, Zakyaei S. Brain death. J Gorgan Uni Med Sci. 2007; 9(2): 5-6.

3) Ronayne C. A phenomenological study to understand the experiences of nurses with regard to brainstem death. Intensive Crit Care Nurs. 2009; 25(2): 90-8. doi: 10.1016/j.iccn.2008.06.001. PMID: 18657425.

4) Matesanz R. A decade of continuous improvement in cadaveric organ donation: the Spanish model. Nefrología. 2001; 21(5): 59-67. PMID: 11881417.

5) Peiffer KM. Brain Death and Organ Procurement: Nursing management of adults with brain injury is crucial to the viability of donor organs. Am J Nurs. 2007; 107(3): 58-67. doi: 10.1097/00000446200703000-00028. PMID: 17314560.

6) Edwards SD, Forbes K. Nursing practice and the definition of human death. Nursing inquiry. 2003; 10(4): 229-35. doi: 10.1046/j.1440-1800.2003.00191.x.

7) Shabanzadeh A, Sadr S, Ghafari A, Nozari B, Toushih M. Organ and tissue donation knowledge among intensive care unit nurses. Transplantation proceedings. Elsevier; 2009.

8) Day L. How nurses shift from care of a brain-injured patient to maintenance of a brain-dead organ donor. Am J Crit Care. 2001; 10(5): 306-12. PMID: 11548563.

9) Ozdag N. The nurses knowledge, awareness and acceptance of tissue-organ donation. Edtna Erca J. 2001; 27(4): 201-6. doi: 10.1111/j.1755-6686.2001.tb00179.x. PMID: 11902635.

10) Nicely B, DeLario GT. Virginia Henderson's principles and practice of nursing applied to organ donation after brain death. Prog Transplant. 2011; 21(1): 72-7. doi: 10.1177/152692481102100110.

11) Coyle MA. Meeting the needs of the family: the role of the specialist nurse in the management of brain death. Intensive Crit Care Nurs. 2000; 16(1): 45-50. doi: 10.1054/iccn.1999.1469. PMID: 10790714.

12) Forsberg A, Flodén A, Lennerling A, Karlsson V, Nilsson M, Fridh I. The core of after death care in relation to organ donation-A grounded theory study. Intensive Crit Care Nurs. 2014; 30(5): 275-82. doi: 10.1016/j.iccn.2014.06.002. PMID: 25042694.

13) Pearson A, Robertson-Malt S, Walsh K, Fitzgerald M. Intensive care nurses' experiences of caring for brain dead organ donor patients. J Clin Nurs. 2001; 10(1): 132-9. doi: 10.1046/j.1365-2702.2001.00447.x. PMID: 11820230. 
14) Broumand B. Transplantation activities in Iran. Exp Clin Transplant. 2005; 3(1): 333 -7. PMID: 15989679.

15) Zohoor AL, Piri Z. Attitudes of Physicians and Nurses of intensive care unit to organ Transplantation with Brain Dead in Hospitals Affiliated with Iran university of Medical Sciences Tehran 2003. RJMS. 2004; 11(39): 97-105.

16) Abbasi Z, Peyman A. Evaluation of brain death and organ transplantation in Iran Medical Law. Iran J Med Law; 2012; 6(20): 43-54.

17) Cebeci F, Sucu G, Karazeybek E. The Roles of Nurses to Augment Organ Donation and Transplantation: A Survey of Nursing Students. Transplantation Proceedings. 2011; 43: 412-4. doi: 10.1016/j.transproceed.2011.01.048. PMID: 21440720.

18) Bernat JL. The concept and practice of brain death. Progress in brain research. 2005;150:369-79. doi: 10.1016/S0079-6123(05)50026-8.

19) Floden A, Berg M, Forsberg A. ICU nurses' perceptions of responsibilities and organisation in relation to organ donation-a phenomenographic study. Intensive Crit Care Nurs. 2011; 27(6): 305-16.

20) Miller AC, Ziad-Miller A, Elamin EM. Brain death and Islam: the interface of religion, culture, history, law, and modern medicine. Clhest. 2014; 146(4): 1092-101. doi: 10.1378/chest.14-0130. PMID: 25287999 PMCID: PMC4188144.

21) Seraji M. The nature of brain death from the viewpoint of Quran and Hadith. Arak Medical University Journal. 2011; 13(5): 65-77.

22) Long T, Sque M, Addington-Hall J. Conflict rationalisation: how family members cope with a diagnosis of brain stem death. Soc Sci Med. 2008; 67(2): 253-61. doi: 10.1016/j.socscimed.2008.03.039. PMID: 18442874.

23) Mirhashemi S. Brain Dead and Transplantion of the Organ of the patient suffering brain Dead. Feghh va hoghoogh khanevade. 2007; 12(44-45): 114-41.

24) Sarbey B. Definitions of death: brain death and what matters in a person. J Law Biosci. 2016; 3(3): 743 -52. doi: 10.1093/jlb/lsw054. PMID: 28852554, PMCID: PMC5570697.

25) DeGrazia D. The Definition of Death. In: Zalta(ed), editor. Stanford Encyclopedia of Philosophy. Stanford University: Metaphysics Research Lab; 2017.

26) Cummiskey D. Declaring Death, Giving Life. Eubios Journal of Asian and International Bioethics. 2005; 15(3): 70-5.

27) Shemie SD, Doig C, Dickens B, Byrne P, Wheelock B, Rocker G, et al. Severe brain injury to neurological determination of death: Canadian forum recommendations. CMAJ. 2006; 174(6): S1-12. doi: 10.1503/cmaj.045142. PMID: 16534069, PMCID: PMC1402399.

28) Salahuddin N, Shafqat S, Mapara S, Khan S, Siddiqui S, Manasia R, et al. End of life in the intensive care unit: knowledge and practice of clinicians from Karachi, Pakistan. Intern Med J. 2008; 38(5): 307-13. doi: 10.1111/j.1445-5994.2007.01595.x. PMID: 18402559.

29) Simpson P, Bates D, Bonner S, Costeloe K, Doyal L, Falvey S, et al. A Code of Practice for the Diagnosis and Confirmation of Death. London: GB Academy of Medical Royal Collages; 2008.

30) Yazdimoghaddam H, Manzari ZS. Training; a golden ring for removing nurses' challenges in caring process of the brain death patients. Hayat. 2017; 23(2): 100-5.

31) Sotoudeh H, Kalhornia Golkar M. Brain Death: From Nature to Juridical-Legal Effects. Medical Law. 2012; 6(22): 113-50.

32) White G. Intensive care nurses' perceptions of brain death. Aust Crit Care. 2003; 16(1): 7-14. doi: 10.1016/S1036-7314(03)80023-1.

33) Keshtkaran Z, Sharif F, Navab E, Gholamzadeh S. Lived Experiences of Iranian Nurses Caring for Brain Death Organ Donor Patients: Caring as "Halo of Ambiguity and Doubt". Glob J Health Sci. 2016; 8(7): 281-92. doi: 10.5539/gjhs.v8n7p281. PMID: 26925919, PMCID: PMC4965685.

34) Marck CH, Weiland TJ, Neate SL, Hickey BB, Jelinek GA. Australian emergency doctors' and nurses' acceptance and knowledge regarding brain death: a national survey. Clinical transplantation. 2012; 26(3): 254-60.

35) Kima JR, Elliotta D, Hyde C. Korean health professionals' attitudes and knowledge toward organ donation and transplantation. Int J Nurs Stud. 2004; 41: 299-307. doi: 10.1016/j.ijnurstu.2003.10.003. PMID: 14967187.

36) Flodén A, Forsberg A. A phenomenographic study of ICU-nurses' perceptions of and attitudes to organ donation and care of potential donors. Intensive Crit Care Nurs. 2009; 25(6): 306-13. doi: 10.1016/j.iccn.2009.06.002. PMID: 19608419. 
37) Cohen J, Ami SB, Ashkenazi T, Singer P. Attitude of health care professionals to brain death: influence on the organ donation process. Clinical transplantation. 2008; 22(2): 211-5. doi: 10.1111/j.13990012.2007.00776.x. PMID: 18339141.

38) Rittner CK, Besold A, Wandel E. A proposal for an anonymous living organ donation in Germany. Legal Medicine. 2003; 5: S68-71. doi: 10.1016/S1344-6223(02)00126-8.

39) Yousefi AR, Ziaee ES, Ghajaee F, Azarbarzin M. Brain death and transplant organs from the perspective of jurisprudence,ethics and law and its impact on medical education. IJME/Special issue for educational development and health promotion. 2012; 11(9): 1366-75.

40) Tan HM, Wilson A, Olver I, Barton C. The experience of palliative patients and their families of a family meeting utilised as an instrument for spiritual and psychosocial care: A qualitative study. BMC Palliat Care. 2011; 10: 7. doi: 10.1186/1472-684X-10-7. PMID: 21435259, PMCID: PMC3073937. 